Available online at www.banglajol.info

Bangladesh J. Sci. Ind. Res. 44(1), 31-40, 2009
BANGLADESH JOURNAL OF SCIENTIFIC AND INDUSTRIAL RESEARCH

E-mail: bjsir07@gmail.com

\title{
Studies on Black Iron Oxide Pigment. Part-II: Effect of Preparation Parameter on Particle Size and Microstructure of Ferrosoferric Oxide
}

\author{
A.J.M. Tahuran Negera*, Aftabuddin Ahmeda, Sufia Parvina and A.M. Shafiqul Alamb \\ aIGCRT, BCSIR, Dhaka-1205 and bDepartment of Chemistry, \\ Dhaka University, Dhaka, Bangladesh
}

\begin{abstract}
This paper deals with the effect of preparation parameter on particle size and microstructure of the prepared ferrosoferric oxide. 10 prepared samples (from 23 samples) and one standard sample (Bayferrox 318 standard 88) collected from BAYER, Germany was selected for this study. X-ray diffraction, petrographic microscopy, scanning electron microscope and particle size analyzer were used to characterize the phase present, particle size and shape of the particle. It is observed from X-ray diffraction that all these samples mainly contain $\mathrm{Fe}_{3} \mathrm{O}_{4}$ phase. The shape of the particle is found to be round and agglomerated. The average particle diameter of highest portion of pigment in the peak region is ranged from $12.25 \mu$ to $17.32 \mu$, which is very similar to the standard sample (17.32 $\mu)$. Sample number 9 , which was prepared with ferrous sulphate, hydrochloric acid, sodium nitrate and ammonium hydroxide at a molar ratio 1:1.74:0.16:4.07 and standard sample contain narrow high peak in their particle size distribution curve. Another observation is that, experimental parameter, though have some effect on particle size but have no effect on microstructure.
\end{abstract}

Key words: Black iron oxide, Pigment, Particle size, Microstructure, Ferrosoferric oxide.

\section{Introduction}

This work is a continuation of a study on black iron oxide pigment (Neger et.al.2008). In that paper, effect of preparation parameter on the physical and optical properties as well as the statistical evaluation on the preparative methods was investigated. Particle size affects surface characteristics such as smoothness, brightness and uniformity when the pigments are incorporated into media to make paints. The most important physical property of a pigment is hiding power, which also depends on particle shape and hence upon the method of manufacture. Again, opacity can be increased by increasing their surface area as well as by reducing the size of the particle. Other size dependent proper-

* Corresponding author. 
ties of pigments are plasticity, durability and gloss.

Although many studies have been done on the preparative method of ferrosoferric oxide (Ayers and Joseph 1939, Kensavgya et. al. 1963, Dobos et. al. 1963, Frey and Friedrich 1962, Farbenind 1936, Maruschek and Hans 1959, Manfred and August 1970, Reymer 1956, Srivastava et.al. 1959, Szigeti, 1961, Toma et. al. 1964, Williams et. al. 1941, Yoshiro (1958), but there is only very scanty information on microstructure of these pigment. The objective of the present study is to evaluate the effect of preparation parameter on particle size and their microstructure.

\section{Materials and Methods}

\section{Materials}

Commercial grade materials were used through out the study. The chemicals required for the above purpose were ferrous sulphate heptahydrate (97.87\%), concentrated hydrochloric acid (32\%), ammonium hydroxide (17\%), sodium hydroxide, sodium carbonate, calcium hydroxide and sodium nitrate.

\section{Preparation}

Depending upon the reaction parameter, 10 out of the 23 prepared samples and one reference sample were selected for the present investigation. The samples were so selected that effects of all important preparation parameters are represented. These samples were prepared by conversion of ferrous sulphate to ferrous chloride followed by the treatment of precipitating agent in presence of an oxidizing agent. Air was passed throughout the system constantly at the rate of $20 \mathrm{lb} / \mathrm{min}$. The speed of stirring, the capacity of the reaction vessel and the temperature of the reaction media were kept constant (500rpm, 5L and 90C respectively) for all the experiments. The experimental conditions which were varied for the preparation of these samples are; for sample-1, molar ratio of iron to hydrochloric acid, sodium nitrate and ammonia as precipitating agent was 1:0.5:0.16:3.0. For sample-2, the molar ratio was same but the precipitating agent was sodium carbonate, for sample-3, the precipitating agent was sodium hydroxide and for sample-4, that was calcium hydroxide. For sample number 5 and 6, the molar ratio of iron to hydrochloric acid, sodium nitrate and ammonium hydroxide were 1:0.75:0.16:3.0 and 1:1.74:0.16:3.0 respectively. Sample number 7, 8 and 9 were prepared by changing molar ratio of iron to ammonium hydroxide only. The conditions of reaction parameters for these three samples were molar ratio of iron to hydrochloric acid, sodium nitrate and ammonium hydroxide 1:1.74:0.16:2.71, 1:1.74:0.16:3.39 and 1:1.74:0.16:4.07 respectively. The ratio of hydrochloric acid and oxidizing agent with iron of sample number 1 was kept constant for these three experiments. And sample number 10 was prepared by altering the 
molar ratio of iron to oxidizing agent keeping all other reaction parameter of sample number 9 constant. The condition of reaction parameter of sample number 10 was molar ratio of iron to hydrochloric acid, sodium nitrate and ammonium hydroxide 1:1.74:0.16:2.71.

\section{Testing procedure}

\section{Particle size}

Particle size of these pigments was determined by a 'Micromeritics particle size analyzer of model 5100' using sedimentation method. Reynolds number, liquid density, liquid viscosity and temperature were 0.27 , 0.9939 g.cm $^{-3}, 0.7154 \mathrm{CP}$ and $35.5^{\circ} \mathrm{C}$ respectively and kept constant throughout the experiments. Mean and standard deviation on particle size of these pigments were calculated by statistical method (Mostafa 1984.

\section{External surface area}

The external surface area of powdered and granular materials can be calculated (Hilton 1968) from particle size distribution measurements,

$$
S_{\mathrm{w}}=\frac{\alpha}{\rho} \Sigma \frac{\mathrm{W}_{\mathrm{i}}}{\mathrm{M}_{\mathrm{i}}}
$$

Where $\alpha$ is a shape factor that equals 6 for spherical or hereby spherical particles, $\rho$ is the density, $\mathrm{W}_{\mathrm{i}}$ is the weight fraction per unit weight and $\mathrm{M}_{\mathrm{i}}$ is the mean particle size of the corresponding size interval. If $r$ is expressed in $\mathrm{g} / \mathrm{cm}^{3}$ and $\mathrm{Mi}$ in $\mathrm{cm}$, then $\mathrm{Sw}$ is in $\mathrm{cm}^{2} / \mathrm{g}$.

\section{X-ray diffraction studies}

The X-ray diffraction patterns were recorded by an X-ray difractometer (Model JDX-8P JEOL) using $\mathrm{Zr}$ filter and MoK $\alpha$ radiation in the Department of Metallurgical Engineering, Bangladesh University of Engineering and Technology. The patterns were obtained between two-theta range of $5-60^{\circ}$ at $30 \mathrm{KV}$ and $20 \mathrm{~mA}$. A scanning speed of $20 / \mathrm{min}$ was used.

\section{Photomicrograph}

Photomicrographs of black iron oxide pigment of sample 1 and 7 were done by a petrographic microscopy of model LEICA DM LP attached with MPS 30 camera.

\section{Scanning electron micrograph}

SEM of black iron oxide pigment (sample 7) was done at the Department of Material Science and Engineering, Ohio State University, U.S.A. Prior to observation sample was gold coated. EDS analysis was carried out at the same time.

\section{Results and Discussion}

The most significant size for pigments is the projected area diameter since this diameter governs the covering power of the pigment. The objective of this study is to observe how preparation parameters affect the particle size and microstructure of the said pigments. Table I showed the mass percent of average 


\begin{tabular}{|c|c|c|c|c|c|c|c|c|c|c|c|c|c|c|c|c|c|c|c|c|c|c|}
\hline & & 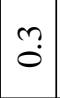 & $\mid$\begin{tabular}{l|}
$\infty$ \\
0 \\
0
\end{tabular} & $\begin{array}{l}0 \\
0 \\
0\end{array}$ & $\stackrel{\vec{i}}{\stackrel{\nabla}{*}}$ & $\begin{array}{l}0 \\
0\end{array}$ & $\begin{array}{l}0 \\
0\end{array}$ & $\stackrel{1}{0}$ & $\begin{array}{l}\tilde{1} \\
0\end{array}$ & $\mid \begin{array}{l}0 \\
0\end{array}$ & $\begin{array}{l}n \\
0 \\
0\end{array}$ & $\overrightarrow{0}$ & $\stackrel{n}{0}$ & $\stackrel{3}{-}$ & $\stackrel{-1}{-}$ & 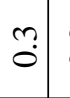 & 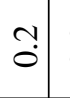 & $\begin{array}{llll}0 & 0 \\
0 & 0\end{array}$ & $\begin{array}{lll}0 & 0 \\
0 & 0\end{array}$ & $\stackrel{9}{-i}$ & Oे & $\stackrel{0}{0}$ \\
\hline & & & $\mid$ & $\mid \begin{array}{l}\stackrel{i}{i} \\
\end{array}$ & $\tilde{i}$ & $\because$ & $\begin{array}{l}0 \\
0\end{array}$ & $\stackrel{\nabla}{0}$ & $\stackrel{+}{0}$ & \begin{tabular}{|c|}
$\infty$ \\
0 \\
0
\end{tabular} & $\ddot{0}$ & 0 & 它 & $\stackrel{\nabla}{\sim}$ & $\begin{array}{l}\infty \\
i \\
i\end{array}$ & பִ. & 3 & \begin{tabular}{l|l}
0 \\
0 & 0
\end{tabular} & \begin{tabular}{lll}
0 & $\Omega$ \\
\hdashline & -
\end{tabular} & $\stackrel{\infty}{-}$ & $\stackrel{0}{0}$ & $\dot{0}$ \\
\hline & & & $\mid \begin{array}{l}\infty \\
0 \\
0\end{array}$ & $\mid \begin{array}{l}\infty \\
\dot{\forall} \\
+\end{array}$ & $\vec{i}$ & $\stackrel{0}{0}$ & $\mid$\begin{tabular}{l|}
0 \\
0
\end{tabular} & $\mid$\begin{tabular}{l|}
$\infty$ \\
0 \\
0
\end{tabular} & $\stackrel{\sim}{\sim}$ & 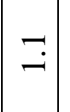 & $\stackrel{n}{\sim}$ & 占 & $\stackrel{m}{-}$ & ஸึ่ & $\stackrel{m}{i}$ & $\stackrel{\infty}{0}$ & $\stackrel{m}{0}$ & \begin{tabular}{l|l}
0 & 1 \\
0 & 0
\end{tabular} & \begin{tabular}{l|l}
$\stackrel{\sim}{O}$ & $\hat{m}$
\end{tabular} & ब. & $\begin{array}{l}0 \\
0\end{array}$ & $\stackrel{\circ}{\circ}$ \\
\hline & & & -1 & $\mid \begin{array}{l}0 \\
\dot{0}\end{array}$ & $\stackrel{\sim}{\sim}$ & $\stackrel{\sim}{-}$ & $\mid \begin{array}{l}n \\
0 \\
0\end{array}$ & $\begin{array}{l}\stackrel{0}{i} \\
\stackrel{i}{*}\end{array}$ & $\stackrel{m}{-}$ & $\stackrel{m}{\sim}$ & $\exists$ & $\overrightarrow{\dot{m}}$ & $\stackrel{+}{-}$ & مִ & $\widehat{i}$ & $\stackrel{-1}{-}$ & $\stackrel{m}{-}$ & \begin{tabular}{l|l}
$L$ \\
0 \\
0
\end{tabular} & \begin{tabular}{c|c|c}
0 \\
$\dot{0}$
\end{tabular} & $\stackrel{9}{-1}$ & $\overrightarrow{0}$ & $\stackrel{0}{\circ}$ \\
\hline & & & $\begin{array}{l}a \\
7\end{array}$ & $\dot{\sigma}$ & $\begin{array}{c}n \\
\infty \\
\infty\end{array}$ & $\stackrel{\overbrace{}}{-i}$ & O. & $\left|\begin{array}{l}m \\
m\end{array}\right|$ & $\stackrel{+}{\dot{m}}$ & $\mid \begin{array}{r}+ \\
\dot{m}\end{array}$ & $\stackrel{\circ}{\mathrm{N}}$ & $\underset{\forall}{+}$ & $\stackrel{b}{\sim}$ & ָ̃. & 0 & $\stackrel{\nabla}{\sim}$ & $\stackrel{0}{\sim}$ & 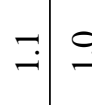 & مִ & $\begin{array}{l}\infty \\
\dot{m}\end{array}$ & 3. & 3 \\
\hline & & & $\mid \begin{array}{l}0 \\
\infty \\
\infty \\
0\end{array}$ & $\left|\begin{array}{c}0 \\
\end{array}\right|$ & $\mid \begin{array}{l}\stackrel{n}{0} \\
\stackrel{M}{\sim}\end{array}$ & $\hat{\mathrm{i}}$ & \begin{tabular}{|l|} 
\\
$m$ \\
$m$
\end{tabular} & $\hat{\theta}$ & $\begin{array}{l}\overrightarrow{0} \\
\dot{0}\end{array}$ & 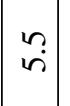 & $\mid \begin{array}{l}0 \\
\stackrel{N}{*}\end{array}$ & -1 & $\begin{array}{l}\dot{m} \\
\dot{m}\end{array}$ & 岂 & 7 & ㅇ. & $\stackrel{m}{n}$ & $\vec{i}$ & $\stackrel{n}{=}$ & $\stackrel{9}{+}$ & $\because$ & ָ̃ \\
\hline & & & 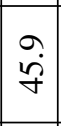 & 芦 & 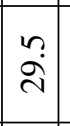 & ṽ & $\begin{array}{l}m \\
\dot{m} \\
\dot{m}\end{array}$ & $\overrightarrow{\dot{m}}$ & $\begin{array}{l}0 \\
\dot{i} \\
\stackrel{1}{n}\end{array}$ & -1 & 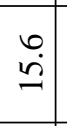 & 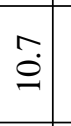 & $\curvearrowright$ & $\begin{array}{l}0 \\
\stackrel{N}{\mathrm{~N}}\end{array}$ & $\begin{array}{l}0 \\
\stackrel{0}{0}\end{array}$ & $\infty$ & $\underset{n}{n}$ & $\stackrel{\vec{r}}{\vec{\sigma}}$ & 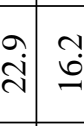 & $\begin{array}{l}\circ \\
\dot{a}\end{array}$ & $\stackrel{m}{0}$ & $\stackrel{\circ}{\dot{m}}$ \\
\hline & & ఠ & $\stackrel{N}{n}$ & $\mid$\begin{tabular}{l|}
0 \\
$\dot{0}$
\end{tabular} & $\begin{array}{l}\stackrel{0}{+} \\
\dot{\mathbb{U}}\end{array}$ & 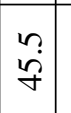 & $\left|\begin{array}{l}\overrightarrow{+} \\
\dot{m}\end{array}\right|$ & $\begin{array}{l}\hat{m} \\
\dot{m}\end{array}$ & $\begin{array}{l}\infty \\
\stackrel{\infty}{\mid} \\
\stackrel{n}{n}\end{array}$ & $\hat{\tilde{\nu}}$ & 号 & t. & $\begin{array}{l}\infty \\
\dot{\sim} \\
\dot{\sim}\end{array}$ & $\begin{array}{l}0 \\
\text { } \\
\tilde{m}\end{array}$ & 임 & $\begin{array}{l}0 \\
\dot{\sim}\end{array}$ & $\underset{ \pm}{+}$ & $\begin{array}{c}\stackrel{\sim}{\nu} \\
\stackrel{\sim}{\sim}\end{array}$ & 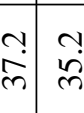 & 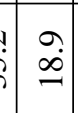 & m. & $\stackrel{\text { ㄱ. }}{\text { f }}$ \\
\hline$y$ & & 0 & $\ddot{0}$ & $\underset{⿱ 亠 乂}{\Sigma}$ & $\begin{array}{l}0 \\
0\end{array}$ & $\begin{array}{l} \\
8 \\
\Phi \\
\grave{2} \\
\end{array}$ & $\mid \begin{array}{l}0 \\
0 \\
0 \\
-1\end{array}$ & \begin{tabular}{|l|} 
\\
$\stackrel{2}{9}$ \\
$\dot{q}$
\end{tabular} & $\begin{array}{l}\sigma \\
\dot{g} \\
-1\end{array}$ & $\mid \begin{array}{c}\mathfrak{N} \\
\dot{q}\end{array}$ & $\begin{array}{l}\hat{\omega} \\
\dot{\theta}\end{array}$ & $\begin{array}{c}\sim \\
\tilde{\omega} \\
\llcorner\end{array}$ & $\begin{array}{l}\infty \\
\dot{\varphi} \\
-1\end{array}$ & $\begin{array}{l}0 \\
\hat{m}\end{array}$ & $\stackrel{0}{\dot{\varphi}}$ & $\begin{array}{l}\hat{\infty} \\
\stackrel{m}{0}\end{array}$ & $\begin{array}{l}0 \\
\stackrel{0}{0}\end{array}$ & \begin{tabular}{l|l}
$n$ & \\
$\tilde{\sigma}$ & $\infty$
\end{tabular} & 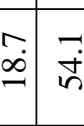 & $\begin{array}{l}0 \\
\dot{m} \\
\end{array}$ & $\begin{array}{c}0 \\
\stackrel{\sim}{\wedge}\end{array}$ & $\stackrel{\sim}{\mathscr{n}}$ \\
\hline$\delta$ & & $\bar{a}$ & $\overrightarrow{0}$ & $\begin{array}{c}2 \\
\infty \\
\infty\end{array}$ & $\begin{array}{l}\mid \\
\tilde{m}\end{array}$ & $\begin{array}{l}\infty \\
\dot{8} \\
\dot{8}\end{array}$ & $\widehat{\mathrm{v}}$ & \begin{tabular}{|c|}
$\dot{0}$ \\
$\dot{\theta}$
\end{tabular} & $\stackrel{ナ}{\stackrel{亠}{*}}$ & $\left|\begin{array}{l}\sigma \\
\dot{\sigma}\end{array}\right|$ & $\ddot{\sigma}$ & 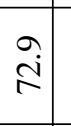 & $\infty$ & $\begin{array}{l}\mathscr{0} \\
\ddot{\vartheta}\end{array}$ & חె & $\stackrel{\infty}{\mathfrak{q}}$ & $\begin{array}{l}\infty \\
\dot{\nu} \\
\dot{\nu}\end{array}$ & \begin{tabular}{c|c} 
& $\nabla$ \\
$\infty$ & 0
\end{tabular} & \begin{tabular}{l|l}
$\nabla$ & 0 \\
$\dot{0}$ & $\infty$ \\
0
\end{tabular} & 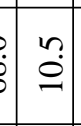 & 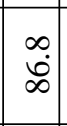 & $\vec{m}$ \\
\hline & & $\begin{array}{l}1 \\
\infty \\
\infty\end{array}$ & $\stackrel{\nabla}{-}$ & $\mid \begin{array}{l}n \\
\grave{0} \\
\infty\end{array}$ & $\begin{array}{l}0 \\
\dot{m}\end{array}$ & $\begin{array}{l}\dot{v} \\
\dot{a}\end{array}$ & $\begin{array}{l}0 \\
0 \\
-i\end{array}$ & $\mid \begin{array}{l}\infty \\
0 \\
0 \\
\end{array}$ & $\begin{array}{l}\sigma \\
+ \\
+\end{array}$ & $\begin{array}{l}0 \\
\dot{\lambda}\end{array}$ & $\begin{array}{l}\infty \\
\dot{0} \\
\dot{0}\end{array}$ & $\stackrel{-1}{-1}$ & $\overrightarrow{6}$ & $\begin{array}{l}\text { @) } \\
\stackrel{\infty}{+}\end{array}$ & $\stackrel{+}{+}$ & $\begin{array}{l}\stackrel{+}{*} \\
\dot{b}\end{array}$ & $\stackrel{\vec{n}}{\vec{n}}$ & \begin{tabular}{l|l}
0 & \\
$\infty$ & \\
$\infty$ & n
\end{tabular} & 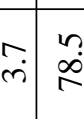 & \begin{tabular}{|l|l|}
0 \\
$\vdots$ \\
$\vdots$
\end{tabular} & $\begin{array}{l}0 \\
\infty \\
\infty\end{array}$ & m. \\
\hline ๙ & & 0 & $\stackrel{+}{\circ}$ & $\left|\begin{array}{l|}1 \\
\infty \\
\infty\end{array}\right|$ & $\begin{array}{l}\infty \\
i \\
\sim\end{array}$ & $\begin{array}{l}\dot{+} \\
\dot{\sigma}\end{array}$ & $\hat{\circ}$ & 点 & $\underset{+}{\stackrel{*}{*}}$ & $\begin{array}{l}\infty \\
\stackrel{1}{\wedge} \\
\end{array}$ & $\overrightarrow{0}$ & ִָ & $\overrightarrow{0}$ & $\begin{array}{c}m \\
\\
\end{array}$ & $\dot{\forall}$ & $\begin{array}{l}\infty \\
\stackrel{1}{0}\end{array}$ & $\dot{m}$ & 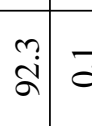 & $\begin{array}{c}-1 \\
0 \\
0\end{array}$ & $\begin{array}{l}\vdots \\
\dot{b}\end{array}$ & नें & $\stackrel{\circ}{\circ}$ \\
\hline & & 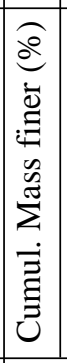 & 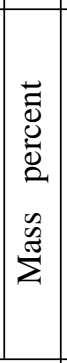 & 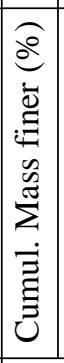 & 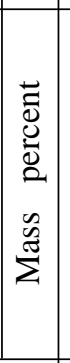 & 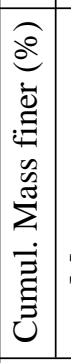 & 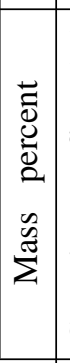 & 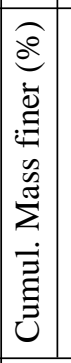 & 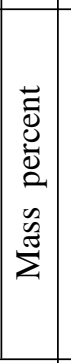 & 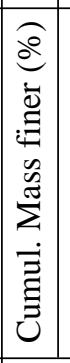 & 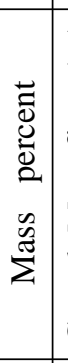 & 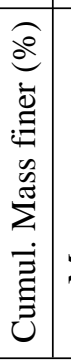 & 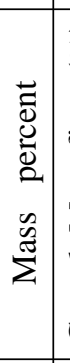 & 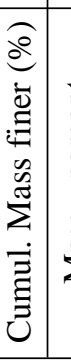 & 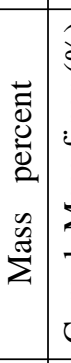 & 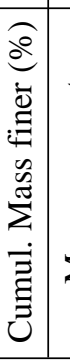 & 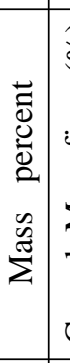 & 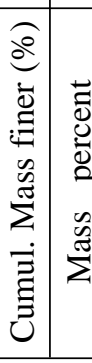 & 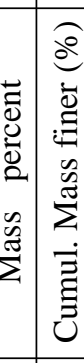 & 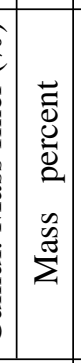 & 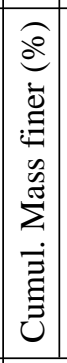 & 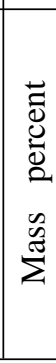 \\
\hline & 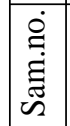 & & & $N$ & & $\mathrm{c}$ & & & & เก & & & & $\Lambda$ & & $\infty$ & & $\sigma$ & & 7 & & \\
\hline
\end{tabular}


particle diameter (from average diameter $2.449 \mu$ to $44.72 \mu$ ) for each pigment. Sample number 1, 2, 3 and 4 were prepared by using different precipitating agent e.g. ammonium hydroxide, sodium hydroxide, sodium carbonate and calcium hydroxide. It is found from this table that sample number 4, which was prepared with calcium hydroxide, is the coarsest and sample number 1 which was prepared with ammonium hydroxide is the finest among these four samples. This indicates that calcium hydroxide, as a precipitating agent make the product particle coarser and ammonium hydroxide as a precipitating agent produces the finest product even than the standard sample (around 9\% sample particle of reference sample has diameter above $44.72 \mu)$. These four products posses the highest portion of pigment at average particle diameter $12.25 \mu$. The reference sample has the highest portion of pigment at average particle diameter $17.32 \mu$. When the products were made by the variation of amount of hydrochloric acid only (sample number 1, 5 and 6), it is found that higher amount of hydrochloric acid used (more than the molar ratio of iron to hydrochloric acid 1:0.5) in the reaction shows negative attitude towards the particle size. Another observation is that amount of ammonium hydroxide as precipitating agent also affects the particle size of the product. It is found that molar ratio of iron to precipitating agent at $1: 4.07$ is the best among the four experiments (1:2.71, 1:3.0, 1:3.39 and 1:4.07). And the last observation from this table is that higher amount of sodium nitrate as oxidizing agent (sample number 1 and 10) than the molar ratio of iron to oxidizing agent 1:0.16 does not show the positive result, and below this ratio, the reaction does not takes place (observed in this laboratory).

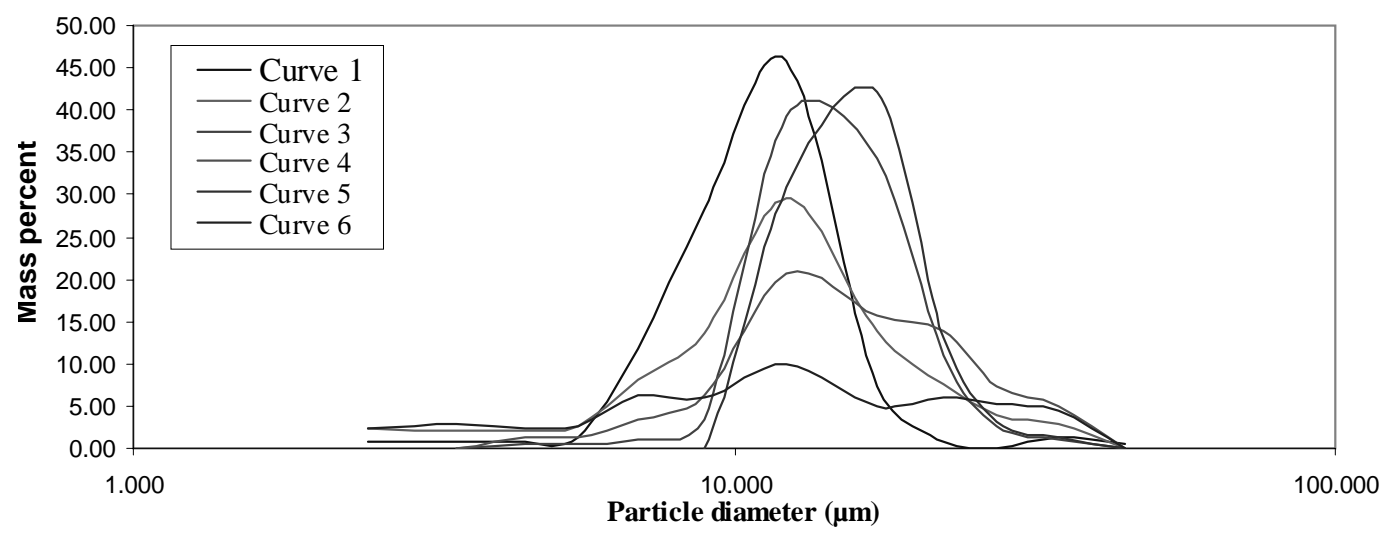

Fig-1: Particle size distribution of some Black Iron Oxide pigment; Curve-1: Sample No-1; Curve-2: Sample No-2; Curve-3: Sample No-3; Curve-4: Sample No-4; Curve-5: Standard Sample and Curve-6: Sample No-7. 
The particle size distributions curves of the samples and reference sample are shown on the figs.1 and 2. These curves are obtained by plotting particle diameters against mass percent. It is known that good pigment properties are observed when most of the particles in the pigment exist in a narrow particle range. This is evident by a sharp peak in the distribution curve. This trend is observed for most of the samples. It is found that particle size distribution of these samples posses only one peak all most at the same region $(12-17 \mu \mathrm{m})$ except sample 4 and 7 . Among them, sample number 1,3 and 9 and reference sample, contain more than 75 percent pigment at the peak region and posses a narrow single peak. When the sample contain below 50 percent pigment at the peak region e.g. sample number 4, 7, 8 and 10, the trend of the curves contain wide single peak or more than one peak. Sample 2, 5 and 6 con- tain more than 50 percent pigment in the peak region. Thus from particle size distribution curve, it can be said that type and amount of precipitating agent during the preparation, affects the trend of particle size distribution curve.

Table II represents the surface area, descriptive statistics on average particle size of some black iron oxide pigment. It is found that sample number 1, 3 and 9 have average particle size higher than that of reference sample (8.308, 7.460 and $9.240 \mu$ respectively). Again sample number 2, 6 and 10 have nearly similar average particle size as standard sample (7.642, 7.275, 70171 and $7.592 \mu$ respectively). All the standard deviations of particle size distribution of black iron pigment are high except sample number 7 and 8. But standard deviation of particle size distribution of sample number $1,3,9$

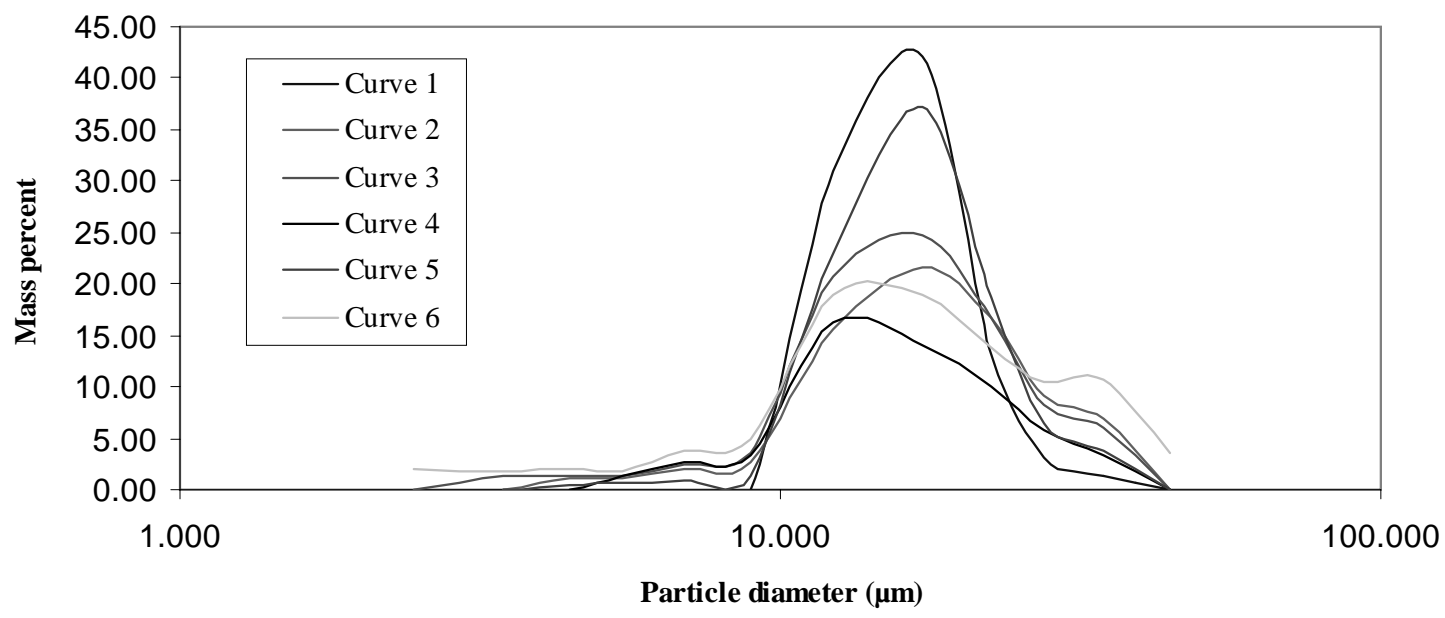

Fig-2: Particle size distribution of some Black Iron Oxide pigment; Curve-1: Standard sample; Curve-2: Sample No-5; Curve-3: Sample No-6; Curve-4: Sample No-8; Curve-5: Sample No-9 and Curve-6: Sample No-10. 
Table II: Descriptive statistics on particle size distribution and surface area of black iron oxide pigment

\begin{tabular}{c|c|c|c}
\hline $\begin{array}{c}\text { No of } \\
\text { Sample }\end{array}$ & $\begin{array}{c}\text { Surface } \\
\text { area }\end{array}$ & $\begin{array}{c}\text { Mean } \\
\text { particle size }\end{array}$ & $\begin{array}{c}\text { Standard } \\
\text { deviation }\end{array}$ \\
\hline 1 & 0.258 & 8.308 & 14.405 \\
2 & 0.203 & 7.642 & 8.124 \\
3 & 0.148 & 9.460 & 14.811 \\
4 & 0.131 & 6.633 & 6.678 \\
5 & 0.134 & 6.433 & 7.571 \\
6 & 0.172 & 7.275 & 8.637 \\
7 & 0.101 & 4.675 & 2.365 \\
8 & 0.147 & 5.067 & 5.489 \\
9 & 0.155 & 9.240 & 12.746 \\
10 & 0.136 & 7.717 & 6.635 \\
Reference & 0.159 & 7.592 & 14.257 \\
Sample & & & \\
\hline
\end{tabular}

and reference sample are extremely high, which spoke that the particle size distribution curves of these samples posses a single sharp peak. Particle size distribution having one or two predominant peak shows better pigment properties than having many peaks. If a pigment has one or two sharp peaks, then the mass percent of that pigment in different region are not so close. For this reason, the standard deviations become high but favorable for particle size of a pigment. The external surface areas of these pigments are also comparable to that of reference sample.

The XRD patterns of sample number 1, 7 and 10 are shown on fig. 3 with their d-values. The patterns were almost completely indexed with JCPDS Powder Diffraction Data File number 26-1136 with the position of the peak and matched very well with those

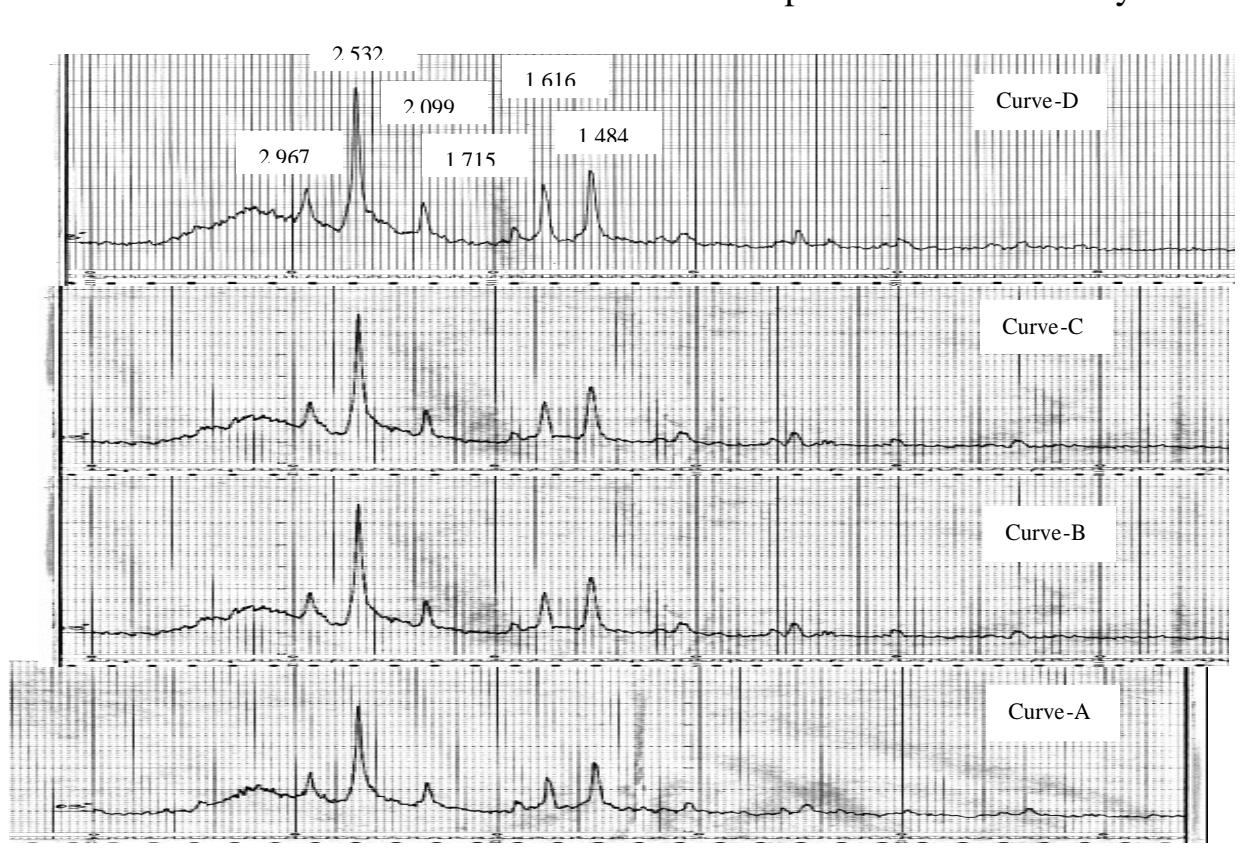

Fig. 3: XRD patterns of some black iron oxide pigment, Curve-A: Standard sample, Curve-B: Sample number-1, Curve-C: Sample number-7 and Curve-D: Sample number-10 
reported for the standards. For the sake of comparison an XRD pattern was also recorded for a reference sample. It can be seen clearly that almost all the peaks matched to those of the reference sample. Indexing is done and within the limits of XRD detection these particular peaks are found to be reflections from (220), (311), (400), (422), (511), (440) and (533) planes. It can be noted that these patterns show the $\mathrm{Fe}_{3} \mathrm{O}_{4}$ phase with

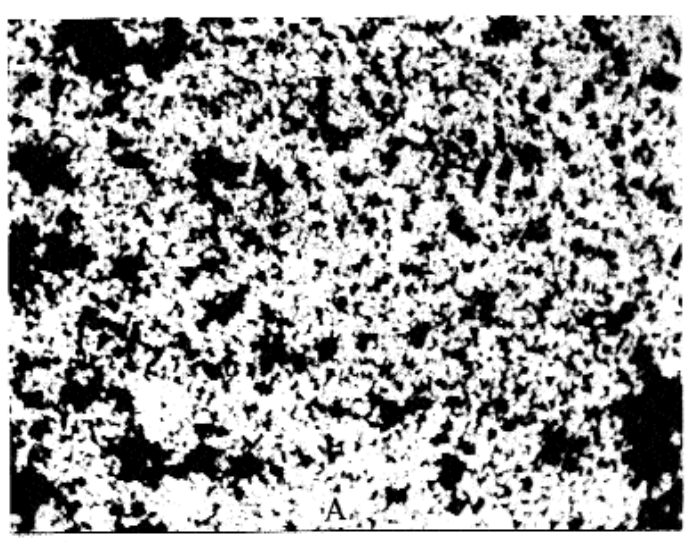

cubic crystal structure having a $=8.0903$. It is also clear from the XRD patterns that the conversion of ferrosoferric oxide from ferrous sulphate heptahydrate in the black iron oxide pigment is completed.

Fig. 4 is a photomicrograph of black iron oxide pigment of sample number 1 and 7 using reflected light in a $100 \mathrm{X}$ magnification. The black area represents the spreaded

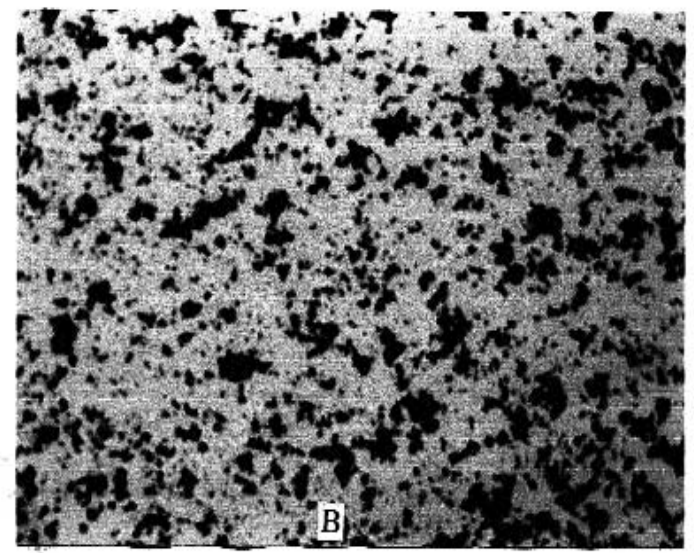

Fig. 4: Photomicrograph of black iron oxide pigment (transmitted light 100X) A: sample number- 1 and $B$ : sample number -7
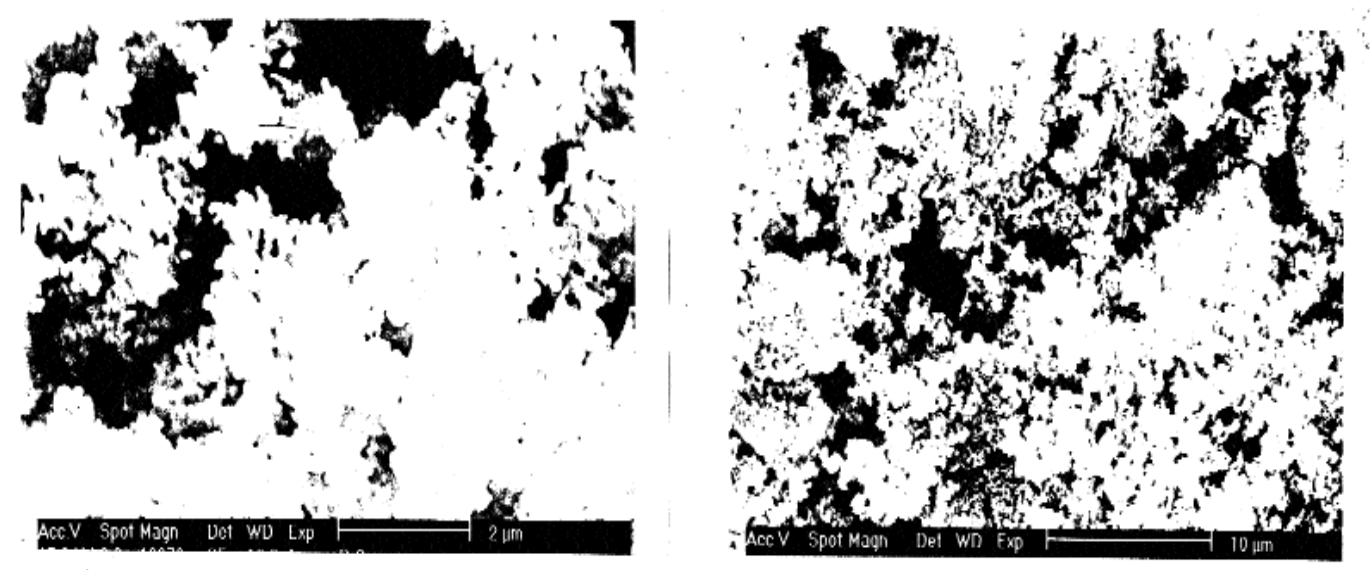

Fig. 5: Scanning electron photomicrograph of black iron oxide pigment (Sample No-7) 


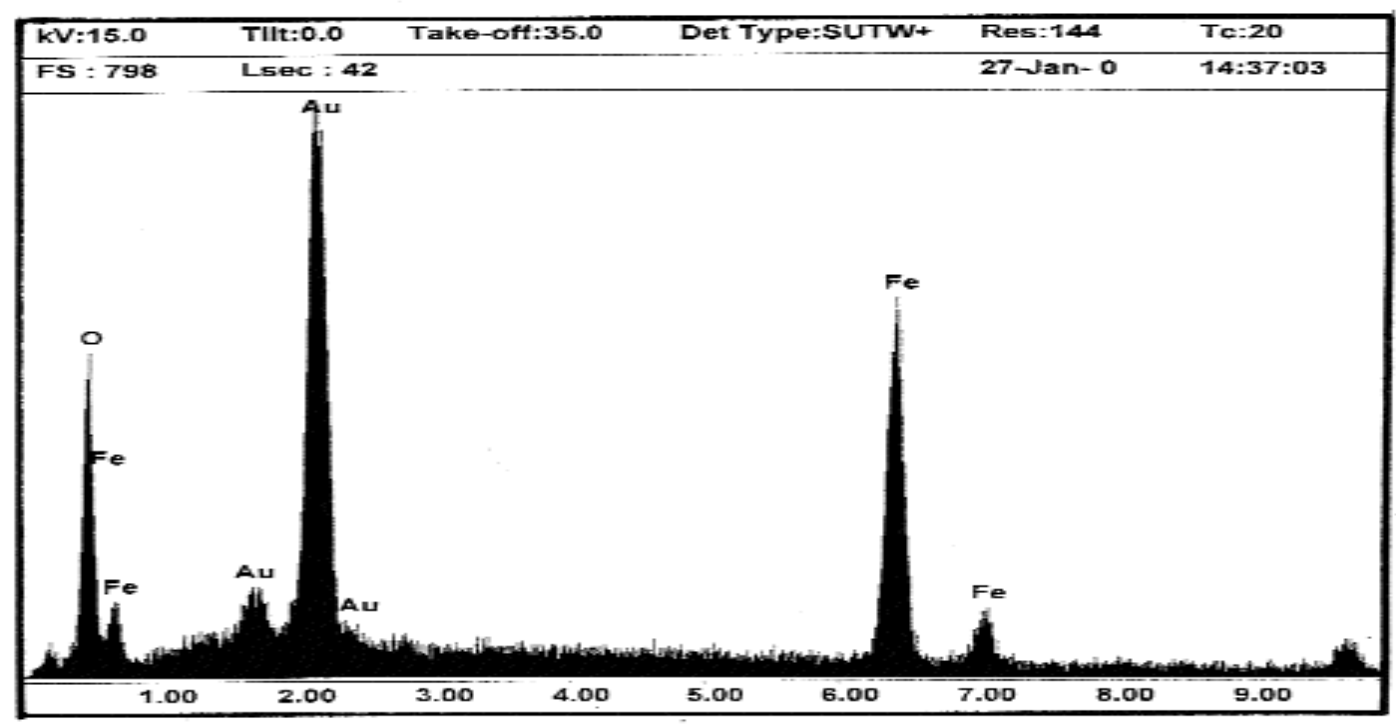

Fig. 6: EDS analysis of sample no 7

pigment. It seems that the shape of particle is spherical. Scanning Electron Micrograph of black iron oxide pigment of sample 7 is shown on figure-5 (magnification $2 \mu$ and $10 \mu)$. It proves the result of photomicrograph and clearly shows that the shape of particle is spherical and agglomerates among themselves. EDS analysis of this sample (fig. 6) confirms the presence of iron and oxygen. Gold peaks come from the Au coating needed for SEM analysis.

\section{Conclusion}

It is found that black iron oxide pigment (sample number 9) prepared from ferrous sulphate heptahydrate using 50ml 32\% hydrochloric acid, 5g sodium nitrate and $300 \mathrm{ml} 17 \%$ ammonia as precipitating agent gave very similar trend of particle size distribution curve with that of reference sample. Reaction parameters though have much effect on the preparation of black iron oxide pigment, but after preparation they all posses $\mathrm{Fe}_{3} \mathrm{O}_{4}$ phase.

\section{Acknowledgements}

The authors express their heartfelt thanks to Md. Nasir Uddin, S.O. Planning and Development Division, BCSIR for helping statistical evaluation on the preparation of this paper on black iron oxide pigment and to Prof. S.A. Akbar, department of Material Science \& Engineering, Ohio State University, Columbus, U.S.A for conducting SEM and XRD discussion and elucidation of phase of the pigment. 


\section{Reference}

Ayers, Joseph W., (1939) Black oxides of iron U.S. Pat. 2, 133,267 (to C.K. Williams \& Co), 18 October 1939; Chem. Abstr, 33: 820.

Dobos, Deszo Kovacs, Jozsef (1963) Production of iron oxide black from ferrous chloride Solution Chem. Abstr. 58 : 14306.

Farbenind, I.G. A-G (1936) Pigments Fr. Pat. March 1936 Chem Abstr. 30: 5435.

Friedrich, F. (1962) Black oxide of iron Br. Pat. 878,679,4 October 1961 Chem. Abstr., 56: 10322.

Hilton, S. (1968) Encyclopedia of industrial chemical analysis, Vol-3, Interscience publishers, Edited by by Foster Dee Snell and clifford \& Hilton, P-67.

Kensavgya, B. (1963) (By Zador, Gyorgy., Baross, Mrs. Jozsef., Kotsis, Endre, Kiss Mis, Erno., \& Almassay, Gyula.) Iron oxide pigment from calcined pyrite Hung. Pat. 149, 523; 15 June 1962; Chem. Abstr., 58: 2176.

Maruschek, Hans K. (1959) Black iron oxide pogment Austrian Pat. 199, 285, 25 August 1958; Chem. Abstr., 53: 5703.

Manfred, S. August, B. (1970), Iron oxide pigments, Germ. Pat. 1,803,637 (to Farbenfabriken Bayer A -G.), 27 May 1970; Chem. Abstr., 73, 2008.

Mostafa, M.G. (1984) Methods of statistics Published By Karim Press And Publica- tion, 3rd Edition 12131415 194-200.

Neger, T.A.J.M. Parvin, S. Ahmed, A. Alam, S.A.M. (2008) Studies on the black iron oxide pigment. Part-1-Effect of preparation parameter on physical and optical pro-perties of ferrosoferric oxide, Bangladesh $J$. Sci. Ind. Res.43(2): 183-196.

Reymers,-Holms Gamla Industri Aktiebolag. (1956) Iron oxide pigment of especially black colot Swed. Pat. 152, 566 (by Holst. T.g. \& Bjornhed K.A.H.); 6 December (1955); Chem. Abstr., 50: 7478.

Srivastava, Krishna C. Kulsrestha, Om P. (1959) Magnetic iron oxides Indian Pat. 61,774 (to Council of Scientific \& Industrial Research), 15 October 1958; Chem, Abstr., 53: 5608.

Szigeti, G. (1961) Manufacture of black iron oxide pigments in the presence of nitrate Chem. Abstr. 55: 3088.

Toma, I. Getia, V. Gusatu, N. (1964) New Method for preparation of black iron oxide by precipitation, Chem. Abstr.,61: 4592.

Williams \& Co., C.K. (1941). Black $\mathrm{Fe}_{3} \mathrm{O}_{4}$ pigments Germ. Pat. 700,939; 5 December 1940, Chem. Abstr. 35: 7739.

Yoshiro, A. (1958). Ferrosoferric oxide Jap. Pat. 9564 (“56); 9 December 1956, Chem. Abstr., 52: 19042.

Received :December, 05, 2007;

Accepted : March, 23, 2008. 